\title{
Radiotherapy for initial clinically positive internal mammary nodes in breast cancer
}

\author{
Jina Kim¹, Jee Suk Chang ${ }^{1}$, Seo Hee Choi ${ }^{1}$ Yong Bae Kim¹, Ki Chang Keum¹, Chang-Ok Suh', Gowoon Yang ${ }^{1}$, \\ Yeona $\mathrm{Cho}^{2}$, Jun Won Kim², Ik Jae Lee ${ }^{2}$ \\ ${ }^{1}$ Department of Radiation Oncology, Yonsei Cancer Center, Yonsei University College of Medicine, Seoul, Korea \\ ${ }^{2}$ Department of Radiation Oncology, Gangnam Severance Hospital, Yonsei University College of Medicine, Seoul, Korea
}

\begin{abstract}
Purpose: Internal mammary lymph node (IMN) involvement is associated with poor prognosis in breast cancer. This study investigated the treatment outcomes of initial clinically IMN-positive breast cancer patients who received adjuvant radiotherapy (RT), including IMN irradiation, following primary breast surgery.

Materials and Methods: We retrospectively reviewed data of 95 breast cancer patients with clinically detected IMNs at diagnosis treated with surgery and RT between June 2009 and December 2015. Patients received adjuvant RT to the whole breast/ chest wall and regional lymph node (axillary, internal mammary, and supraclavicular) areas. Twelve patients received an additional boost to the IMN area.

Results: The median follow-up was 43.2 months (range, 4.5 to 100.5 months). Among 77 patients who received neoadjuvant chemotherapy, $52(67.5 \%)$ showed IMN normalization and 19 (24.6\%) showed a partial response to IMN. There were 3 and 24 cases of IMN failure and any recurrence, respectively. The 5 -year IMN failure-free survival, disease-free survival (DFS), and overall survival (OS) were 96\%, 70\%, and 84\%, respectively. IMN failure-free survival was significantly affected by resection margin status (97.7\% if negative, $87.5 \%$ for close or positive margins; $p=0.009$ ). All three patients with IMN failure had initial IMN size $\geq 1 \mathrm{~cm}$ and did not receive IMN boost irradiation. The median age of the three patients was 31 years, and all had hormone receptor-negative tumors. Conclusion: RT provides excellent IMN control without the support of IMN surgery. Intensity-modulated radiotherapy, including IMN boost for breast cancer patients, is a safe and effective technique for regional lymph node irradiation.
\end{abstract}

Keywords: Breast neoplasms, Internal mammary lymph node, Radiotherapy, Prognosis

\section{Introduction}

The internal mammary lymph node (IMN) is a major lymphatic drainage pathway of the breast, draining about $25 \%$ of breast lymphatics $[1,2]$. The reported incidence of IMN involvement in breast cancer patients is 14\%-33\%, varying by tumor location and axillary lymph node status [1,3-5]. IMN involvement is associated with poor prognosis in breast cancer patients, with reported 10-year survival estimates of $37.3 \%-62.4 \%$ [6-8]. Patients with adverse features such as axillary lymphadenopathy, large tumor size, young age, and high histologic grade, often undergo magnetic resonance

Received 28 January 2019, Revised 07 March 2019, Accepted 20 March 2019.

Correspondence: Ik Jae Lee, Department of Radiation Oncology, Yonsei Cancer Center, Yonsei University College of Medicine, 50-1 Yonsei-ro, Seodaemun-gu, Seoul 03722, Korea. Tel: +82-2-2019-3152, Fax: +82-2-2019-4855, E-mail: ikjae412@yuhs.ac (http://orcid.org/0000-0001-7165-3373)

(c) This is an Open Access article distributed under the terms of the Creative Commons Attribution Non-Commercial License (http://creativecommons.org/ licenses/by-nc/4.0/) which permits unrestricted non-commercial use, distribution, and reproduction in any medium, provided the original work is properly cited.

www.e-roj.org 
imaging (MRI) and positron emission tomography/computed tomography (PET-CT) for staging workup at diagnosis [5]. The widespread use of these modern imaging techniques has increased the clinical detection of positive IMNs at diagnosis [9-11].

Historically, surgical dissection and pathological evaluation of IMN chains were offered for patients receiving extended radical mastectomies [12]. However, this practice declined as less aggressive breast conservation surgeries became more common [5]. Recently, some surgeons have suggested that IMN biopsy provides some clinical benefit [13]. Nonetheless, IMNs are frequently undetected in imaging studies, and their biopsy or excision is associated with additional complications such as vascular injuries, pneumothorax, extended operation time, and poor cosmetic outcomes $[14,15]$.

Currently, data on the long-term treatment outcomes of clinically IMN-positive patients who receive radiotherapy (RT) to the involved area without surgical dissection are scarce. Moreover, whether boost irradiation is beneficial and what dose should be administered remain unknown. Few studies have assessed the clinical outcomes of IMN boost irradiation and the optimal radiation dose to radiologically apparent IMNs at diagnosis in breast cancer patients. This study aimed to review the clinical outcomes of initial clinically IMN-positive breast cancer patients treated with RT at our institution.

\section{Methods and Materials}

\section{Patients}

We retrospectively reviewed data of 95 breast cancer patients with clinically detected IMNs at diagnosis treated with surgery and RT between June 2009 and December 2015. Of these, 95 patients with clinically positive IMN detected either by MRI or PET-CT were included in the study. Patients were excluded from the study if they had an M1 stage at diagnosis, received surgical dissection of IMN, did not receive RT to the regional lymph nodes, or did not complete RT. For each patient, MRIs and PET-CTs performed at diagnosis were reviewed, and the number and size of positive IMNs were recorded. Patients were considered to have positive IMNs on the basis of radiologic reports of pre-treatment MRIs or PET-CTs. IMN size was measured by the longest diameter of enlarged IMN according to a picture archiving and communications system. LN size was measured by MRI or for one patient without MRI, by CT images from PET-CT. Tumor characteristics, including molecular type and histologic grade, were recorded, along with treatment characteristics such as surgery type, resection margin, and systemic treatment type (neoadjuvant, adjuvant chemotherapy, and hormone therapy). Luminal A type was defined as estrogen receptor (ER) and progesterone receptor (PR) positive, HER2 negative, and Ki-67 $<14 \%$. Luminal B was defined as ER positive, HER2 negative, and $\mathrm{Ki}-67 \geq 14 \%$ or PR negative or low, or as ER and/or PR positive, and HER2 positive. Non luminal, HER2 positive was defined as no expression of ER and PR and HER2 positive. Triple negative breast cancer was defined as ER, PR, and HER2 negative. IMN response to neoadjuvant chemotherapy was evaluated by comparing the longest diameters of IMN before and after completing neoadjuvant chemotherapy according to the RECIST 1.0 criteria. Complete response was achieved if lymphadenopathy was resolved after neoadjuvant chemotherapy, whereas partial response was achieved if the longest diameter decreased more than $30 \%$.

\section{Radiotherapy}

Each patient's clinical chart was reviewed to determine the RT field, technique, and schedule. Patients received RT to the breast/chest wall and regional lymph nodes in either conventional or hypofractionated schedules. Planning CT was performed in all patients, and patient immobilization was achieved through the use of customized foam positioners and thermoplastic masks. Patients received RT as either threedimensional conformal radiotherapy (3D CRT) or intensitymodulated radiotherapy (IMRT). The RT planning techniques for 3D CRT included reverse hockey stick, standard tangent and anterior beam, partial wide tangential, and photonelectron mixed fields. The RT planning technique for IMRT included partial two-arc volumetric modulated arc therapy (VMAT). All boost irradiation was administered as sequential boosts in 3D CRT patients and as a simultaneous integrated boost in IMRT patients. In the conventional schedule, most patients received $50.4 \mathrm{~Gy}$ of $\mathrm{RT}$ to the whole breast/chest wall and regional LNs (axillary LNs level I-III, SCL, IMN) in 28 fractions and a sequential boost of 5.4-9 Gy to IMNs in 3-5 fractions. Some received 50 Gy RT to the whole breast/chest wall and regional LNs in 25 fractions and a boost of 10 Gy to IMNs in 5 fractions. In the hypofractionated schedule, patients received 42.56 Gy of RT in 16 fractions to the whole breast/ chest wall and regional LNs and a sequential boost of $10 \mathrm{~Gy}$ to the IMNs in 2 Gy fractional doses. For IMRT patients, 48 Gy of RT in 15 fractions was administered to IMNs, while 40 Gy in 15 fractions was administered to the whole breast/chest wall and regional LNs simultaneously. The decision to prescribe a boost dose for IMNs was at the discretion of the treating physicians. 
Table 1 . Patient and treatment characteristics $(n=95)$

\begin{tabular}{|c|c|}
\hline Characteristic & Value \\
\hline Age (yr) & $46(20-73)$ \\
\hline \multicolumn{2}{|l|}{ Laterality } \\
\hline Left & $49(51.6)$ \\
\hline Right & $46(48.4)$ \\
\hline \multicolumn{2}{|l|}{ Pathology } \\
\hline IDC & $89(93.6)$ \\
\hline Others & $6(6.4)$ \\
\hline \multicolumn{2}{|l|}{ cT } \\
\hline $1-2$ & $60(63.1)$ \\
\hline $3-4$ & 35 (36.9) \\
\hline \multicolumn{2}{|l|}{$\mathrm{cN}$} \\
\hline $2 b$ & $6(6.4)$ \\
\hline $3 b$ & 89 (93.6) \\
\hline \multicolumn{2}{|l|}{ IMN+ diagnosis } \\
\hline $\mathrm{MRI}$ & $19(20.0)$ \\
\hline $\mathrm{PET}+\mathrm{MRI}$ & $76(80.0)$ \\
\hline \multicolumn{2}{|l|}{ Initial IMN+ size $(\mathrm{cm})$} \\
\hline$<1$ & $38(40.0)$ \\
\hline$\geq 1$ & $57(60.0)$ \\
\hline \multicolumn{2}{|l|}{ Histologic grade } \\
\hline 1 or 2 & $49(51.6)$ \\
\hline 3 & $32(33.7)$ \\
\hline Unknown & $14(14.7)$ \\
\hline \multicolumn{2}{|l|}{ Molecular type } \\
\hline Luminal A & $9(9.5)$ \\
\hline Luminal B & $35(36.8)$ \\
\hline Non-luminal, HER2+ & $25(26.3)$ \\
\hline TNBC & $26(27.4)$ \\
\hline \multicolumn{2}{|l|}{ LVI } \\
\hline No & $72(75.8)$ \\
\hline Yes & $23(24.2)$ \\
\hline \multicolumn{2}{|l|}{ Neoadjuvant CTx } \\
\hline No & $18(18.9)$ \\
\hline Yes & $77(81.1)$ \\
\hline \multicolumn{2}{|l|}{ Neoadjuvant CTx regimen } \\
\hline $\mathrm{AC}$ & $3(3.9)$ \\
\hline$A C-T$ & $54(70.1)$ \\
\hline FAC & $4(5.2)$ \\
\hline Others & $16(20.8)$ \\
\hline \multicolumn{2}{|l|}{ Adjuvant CTx regimen } \\
\hline AC-T & $11(37.9)$ \\
\hline FAC & $3(10.3)$ \\
\hline Paclitaxel & $3(10.3)$ \\
\hline Others & $12(41.4)$ \\
\hline \multicolumn{2}{|l|}{ Surgery } \\
\hline $\mathrm{BCS}$ & $22(23.2)$ \\
\hline Mastectomy & 73 (76.8) \\
\hline
\end{tabular}

\begin{tabular}{|c|c|}
\hline \multicolumn{2}{|l|}{ Resection margin } \\
\hline Negative & 87 (91.6) \\
\hline Close or positive & $8(8.4)$ \\
\hline \multicolumn{2}{|l|}{ RT modality } \\
\hline 3D CRT & $67(70.5)$ \\
\hline IMRT & $28(29.5)$ \\
\hline \multicolumn{2}{|l|}{ RT schedule } \\
\hline Conventional & 75 (78.9) \\
\hline Hypofractionated & $20(21.1)$ \\
\hline \multicolumn{2}{|l|}{ IMN boost } \\
\hline No & $83(87.3)$ \\
\hline Yes & $12(12.7)$ \\
\hline \multicolumn{2}{|c|}{ IMN boost dose in EQD2 $(\alpha / \beta=4.0)$} \\
\hline Range & $8.7-14.00$ \\
\hline IOR & $8.7-12.37$ \\
\hline \multicolumn{2}{|c|}{$\begin{array}{l}\text { Values are presented as median (range) or number (\%). } \\
\text { IDC, invasive ductal carcinoma; IMN, internal mammary Iymph } \\
\text { node; MRI, magnetic resonance imaging; PET, positron emission } \\
\text { tomography; HER2, human epidermal growth factor receptor } 2 \\
\text { TNBC, triple-negative breast cancer; LVI, Iymphovascular invasion; } \\
\text { AC, adriamycin-cyclophosphamide; AC-T, adriamycin-cyclophos- } \\
\text { phamide-docetaxel; FAC, fluorouracil-adriamycin-cyclophosph- } \\
\text { mide; BCS, breast-conserving surgery; 3D CRT, three-dimensional } \\
\text { conformal radiotherapy; IMRT, intensity-modulated radiotherapy; } \\
\text { IQR, interquartile range. }\end{array}$} \\
\hline
\end{tabular}

The IMN boost target was delineated as a 3-mm margin to the initial enlarged IMN according to a fusion of the initial MR images with $\mathrm{CT}$ simulation images.

\section{Toxicity}

The electronic medical records of each patient were reviewed to investigate toxicities following IMN irradiation. Adverse skin reactions were graded using the Common Terminology Criteria for Adverse Events version 4.0 from the National Cancer Institute [17]. Grade 1 pulmonary toxicity was defined as pneumonitis detected on imaging studies without any clinical symptoms. Grade 2 pulmonary toxicity was defined as symptomatic pneumonitis.

\section{Analysis}

For all recurrent cases, recurrence site and date were recorded. The primary endpoint was IMN failure-free survival, which was defined as the number of months between the first treatment day and the date of follow-up evaluation when IMN recurrence was first observed. The first treatment day was defined as the start day of neoadjuvant chemotherapy or the operation date for patients who did not receive neoadjuvant chemotherapy. 
Table 2. Tumor characteristics of the IMN non-boost and boost groups

\begin{tabular}{|c|c|c|c|}
\hline & $\begin{array}{l}\text { IMN boost }(-) \\
(n=83)\end{array}$ & $\begin{array}{l}\text { IMN boost }(+) \\
(n=12)\end{array}$ & p-value \\
\hline Age (yr) & & & 0.463 \\
\hline$\leq 40$ & $22(26.5)$ & $2(16.7)$ & \\
\hline$>40$ & $61(73.5)$ & $10(83.3)$ & \\
\hline cT & & & 0.458 \\
\hline $1-2$ & $51(61.4)$ & $9(75.0)$ & \\
\hline $3-4$ & $32(18.6)$ & $3(25.0)$ & \\
\hline Initial IMN size $(\mathrm{cm})$ & & & 0.256 \\
\hline$<1$ & $35(42.2)$ & $3(25.0)$ & \\
\hline$\geq 1$ & $48(57.8)$ & $9(75.0)$ & \\
\hline IMN response to neoadjuvant $\mathrm{CTX}$ & & & 0.095 \\
\hline Down-staging $(+)$ & $54(77.1)$ & $5(71.4)$ & \\
\hline Down-staging (-) & $16(22.9)$ & $2(28.6)$ & \\
\hline LVI & & & 0.131 \\
\hline Yes & $18(21.7)$ & $5(41.7)$ & \\
\hline No & $65(78.3)$ & $7(58.3)$ & \\
\hline Molecular subtype & & & 0.221 \\
\hline Luminal A & $9(10.8)$ & $0(0)$ & \\
\hline Luminal B & $32(38.6)$ & $3(25.0)$ & \\
\hline Non-luminal, HER2+ & $22(26.5)$ & $3(25.0)$ & \\
\hline TNBC & $20(24.1)$ & $6(50.0)$ & \\
\hline Histologic grade & & & 0.421 \\
\hline 1,2 & $44(62.9)$ & $5(45.5)$ & \\
\hline 3 & $26(37.1)$ & $6(54.5)$ & \\
\hline Resection margin & & & 0.271 \\
\hline Negative & 77 (92.8) & $10(83.3)$ & \\
\hline Close or positive & $6(7.2)$ & $2(16.7)$ & \\
\hline
\end{tabular}

Values are presented as number (\%).

IMN, internal mammary node; CTX, chemotherapy; LVI, lymphovascular invasion; TNBC, triple-negative breast cancer.

The secondary endpoints were disease-free survival (DFS) and overall survival (OS). DFS was defined as the number of months between the first treatment day and the date when any recurrence was noted. Kaplan-Meier comparisons were used to estimate IMN failure-free survival, DFS, and OS. All statistical analyses were conducted using IBM SPSS Statistics for Windows, version 23.0 (IBM Corp., Armonk, NY, USA).

\section{Results}

A total of 95 women with a median age of 46 years (range, 20 to 73 years) were included in the study. Patient and tumor characteristics are summarized in Table 1. Of 95 patients, MRI and PET-CT were performed at diagnosis for 92 patients; two patients had initial MR images only, and one patient had an initial PET-CT image only. Nineteen patients (20\%) had initial
IMNs detected only on MRI, and 76 patients (80\%) had IMNs detectable on PET-CT. In total, 66 patients had a single IMN detected, 17 had two IMNs detected, and 12 had more than two enlarged IMNs. IMN biopsy was not routinely performed, and only two patients underwent IMN biopsy during breast surgery. Seventy-seven patients (81\%) received neoadjuvant chemotherapy, and 73 patients (77\%) received mastectomy. No patient underwent surgical excision of IMN. Of those who received neoadjuvant chemotherapy, 52 (67.5\%) showed a complete response of IMN after neoadjuvant chemotherapy and 19 (24.6\%) showed a partial response. All 38 HER2positive patients received trastuzumab for at least 1 year postoperatively. A total of 51 patients received postoperative hormone therapy, including 29 premenopausal patients who received tamoxifen and 22 postmenopausal patients who received letrozole. 
A

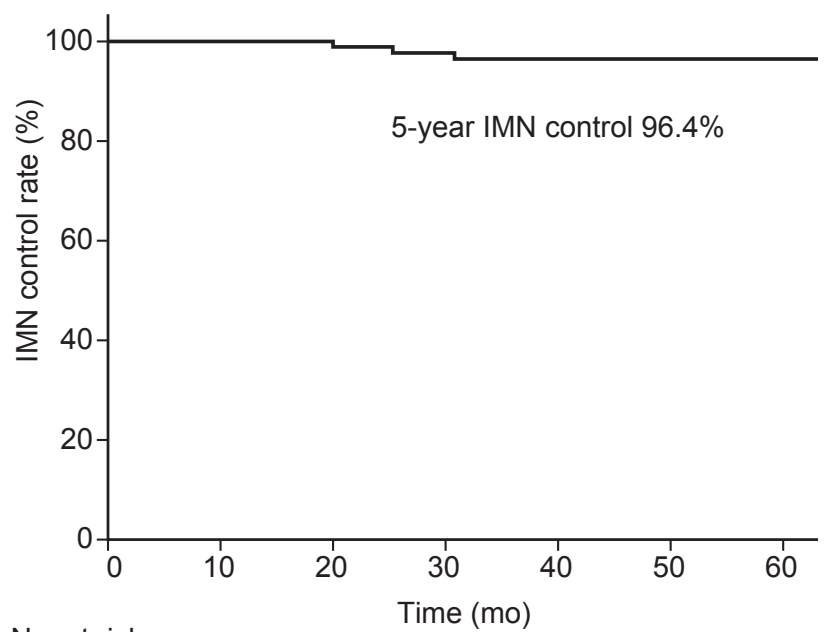

No. at risk

$\begin{array}{lllllll}95 & 94 & 92 & 92 & 92 & 92 & 92\end{array}$
B

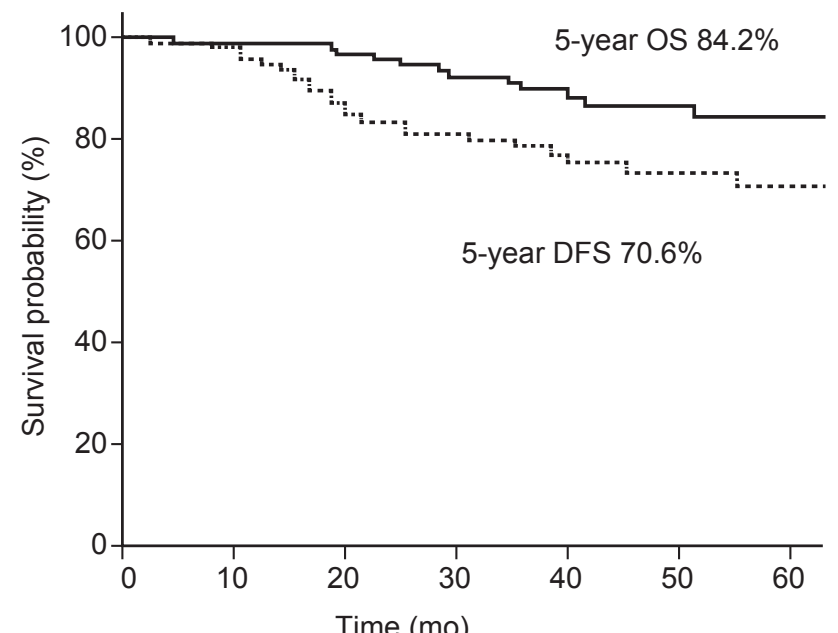

No. at risk

$\begin{array}{lllllll}95 & 93 & 81 & 77 & 73 & 72 & 71 \\ 95 & 90 & 88 & 86 & 85 & 84 & 83\end{array}$

Fig. 1. (A) Kaplan-Meier estimates of IMN-control for the entire cohort and (B) overall survival (OS) and disease-free survival (DFS). IMN, internal mammary node.

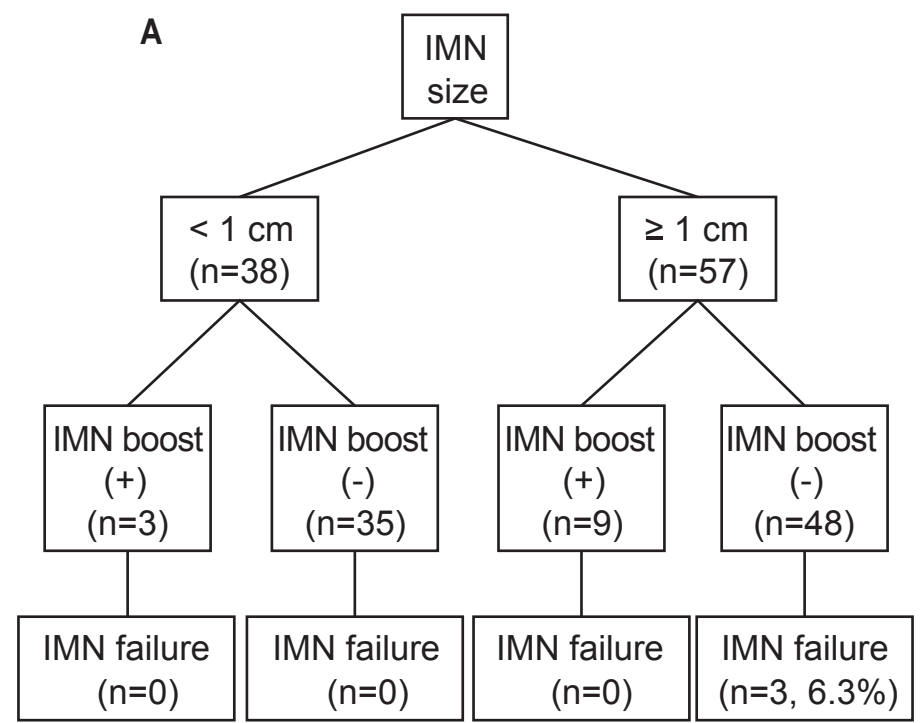

B

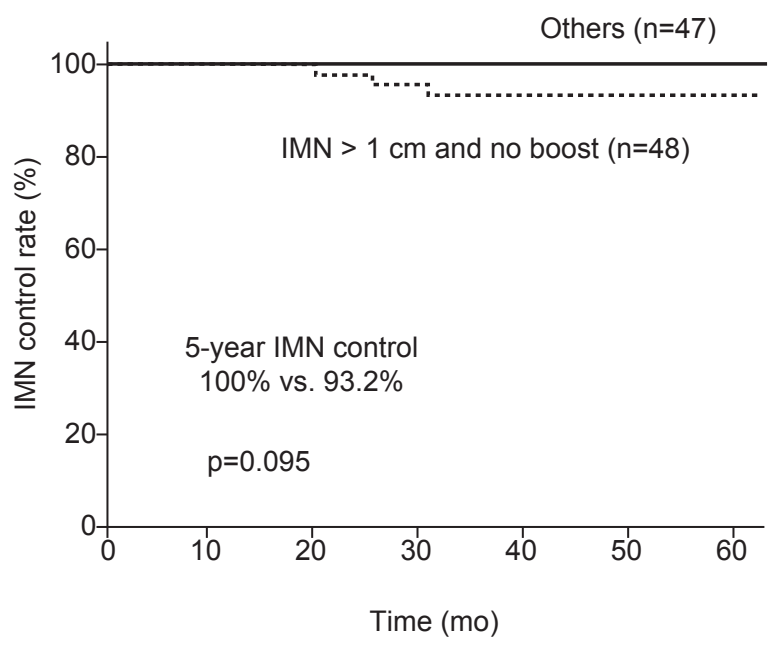

Fig. 2. (A) Patient stratification according to initial IMN size and IMN boost radiotherapy. (B) Kaplan-Meier estimate of IMN-control among patients with initial IMN $\geq 1 \mathrm{~cm}$ without boost RT versus all others. IMN, internal mammary node.

After surgery, all patients received adjuvant $\mathrm{RT}$ to the breast or chest wall and regional lymph nodes including the IMN chain. IMN boost irradiation was performed in 12 patients (12.7\%). No association between IMN response to neoadjuvant chemotherapy and IMN boost RT was noticed in our study. The median IMN boost dose was 10 Gy (interquartile range, 9 to 12 Gy). Sixty-seven patients (70.5\%) were treated with https://doi.org/10.3857/roj.2018.00451
3D CRT and 28 (29.5\%) were treated with IMRT. Patient and tumor characteristics of the boost and non-boost groups did not differ significantly (Table 2). No differences in initial IMN size or response to neoadjuvant chemotherapy were observed between the two groups.

The median follow-up duration from first treatment was 43.2 months (range, 4.5 to 100.5 months). The 5 -year OS 
Table 3. Summary of cases with IMN failure

\begin{tabular}{|c|c|c|c|}
\hline & Case 1 & Case 2 & Case 3 \\
\hline Age (yr) & 55 & 31 & 29 \\
\hline Initial stage & cT3N3b & $\mathrm{cT} 2 \mathrm{~N} 3 \mathrm{~b}$ & cT2N3b \\
\hline Initial IMN (cm) & One, $1.6 \mathrm{~cm}$ & One, $1.1 \mathrm{~cm}$ & One, $1 \mathrm{~cm}$ \\
\hline Neoadjuvant CTx & AC-T \#4 & AC-T \#4 & AC-T \#4 \\
\hline IMN response to $\mathrm{CTX}$ & No visible IMN & No visible IMN & $0.4 \mathrm{~cm}$ IMN \\
\hline Surgery & MRM c ALND & MRM c ALND & MRM c ALND \\
\hline yp stage & ypTONO & ypT1N1a & ypT2NO \\
\hline Resection margin & Negative & Negative & Close \\
\hline Molecular type & Lum B, HER2+ & TNBC & TNBC \\
\hline Adjuvant CTx/HTx & Adjuvant Herceptin & None & None \\
\hline Radiotherapy & $\begin{array}{c}\text { Chest wall, RNI } 50 \text { Gy } \\
\text { without boost }\end{array}$ & $\begin{array}{c}\text { Chest wall, RNI } 40 \text { Gy } \\
\text { without boost }\end{array}$ & $\begin{array}{c}\text { Chest wall, RNI } 50 \text { Gy } \\
\text { without boost }\end{array}$ \\
\hline Recurrence site & IMN & $\begin{array}{c}\mathrm{SCL}, \mathrm{AXL}, \mathrm{IMN}, \mathrm{Rt} \text {. paratracheal, } \\
\text { para-aortic, AP window LNs }\end{array}$ & $\begin{array}{c}\mathrm{SCL}, \mathrm{AXL} \text {, IMN, neck level IV-VI, } \\
\text { T7 spine, sternum, liver }\end{array}$ \\
\hline DFS (mo) & 30.7 & 19.9 & 25.3 \\
\hline OS (mo) & 87.1 & 36.9 & 28.4 \\
\hline
\end{tabular}

IMN, internal mammary node; CTx, chemotherapy; AC-T, adriamycin, cyclophosphamide-paclitaxel; MRM, modified radical mastectomy; ALND, axillary lymph node dissection; TNBC, triple-negative breast cancer; HTx, hormone therapy; RNI, regional lymph node irradiation; $\mathrm{SCL}$, supraclavicular lymph node; $\mathrm{AXL}$, axillary lymph node; $\mathrm{AP}$ window, aortopulmonary window; LN, lymph node; DFS, disease-free survival; OS, overall survival.

was $84.2 \%$, and the 5 -year DFS was $70.6 \%$, with 24 cases of any recurrence reported (Fig. 1). Among 24 cases with any recurrence, six patients had locoregional failure, 14 had distant metastases, and 4 had both locoregional and distant metastases. Only 2 of the 10 locoregional failure cases were noted on the chest wall, with the remaining cases experiencing recurrence in the regional LN areas. Factors related to DFS included initial clinical T stage, IMN response to neoadjuvant chemotherapy, lymphovascular invasion, and resection margin. IMN boost was not significantly associated with DFS. The 5-year IMN control probability was 96.4\%, and no IMN failure was noted in the IMN boost-irradiated group. IMN failures were first noted on routine MRIs, and were confirmed through PET-CTs. The three cases of IMN failure noted in this study are summarized in Table 3.

In the univariate analysis (Table 4), IMN control was significantly associated with close $(<2 \mathrm{~mm})$ or positive resection margin $(p=0.009)$. Other factors, including age, tumor location, clinical T stage, initial IMN size or number, IMN response to neoadjuvant chemotherapy, Iymphovascular invasion, molecular subtype, and histologic grade, were not significantly associated with IMN control. IMN control rates, stratified by initial IMN size and IMN boost, are shown in Fig. 2. Patients with initial IMN size $\geq 1 \mathrm{~cm}$ and who did not receive IMN boost irradiation had poorer 5-year IMN control, but the difference was not statistically significant (100\% vs. 93.2\%; $p=0.095)$. No difference in prognosis was observed between the 3D CRT and IMRT groups or between the conventional and hypofractionated schedule groups (data not shown).

No adverse effects of grade 3 or higher were reported in this study. Higher radiologic lung toxicity (37.5\% vs. 5.1\%; $p$ $=0.003$ ) and mild skin reaction (50.0\% vs. $15.3 \% ; p=0.020)$ were observed in the IMN boost-irradiated patients, but only in the 3D CRT group. No adverse reaction of any kind was reported following IMN boost with IMRT. No patient presented with cardiac toxicity of any grade following RT, and severe treatment-related toxicity was not observed regardless of IMN boost.

\section{Discussion and Conclusion}

Management of the IMN chain in breast cancer patients with or without IMN involvement remains controversial, as detection and surgical approaches are difficult owing to the location. Yet IMN should not be neglected because IMN involvement and under-treatment are associated with a poor prognosis [18]. Surgical dissection of IMN has been actively performed in the past but was abandoned due to lack of survival benefit and high potential risk for morbidity $[4,19]$ However, with modern imaging modalities such as MRI and 
Table 4. Prognostic factors related to IMN control

\begin{tabular}{|c|c|c|c|}
\hline & \multirow{2}{*}{ No. of patients $(\%)$} & \multicolumn{2}{|c|}{ Univariate } \\
\hline & & $5-y r$ rate $(\%)$ & $p$-value \\
\hline Age (yr) & & & 0.132 \\
\hline$\leq 40$ & $24(25.3)$ & 91.7 & \\
\hline$>40$ & $71(74.7)$ & 98.1 & \\
\hline cT & & & 0.942 \\
\hline $1-2$ & $60(63.2)$ & 96.5 & \\
\hline $3-4$ & $35(36.8)$ & 96.3 & \\
\hline Initial IMN size $(\mathrm{cm})$ & & & 0.156 \\
\hline$<1$ & $38(40.0)$ & 100 & \\
\hline$\geq 1$ & $57(60.0)$ & 94.1 & \\
\hline IMN response to neoadjuvant CTx & & & 0.407 \\
\hline Down-staging $(+)$ & $59(76.6)$ & 94.6 & \\
\hline Down-staging (-) & $18(23.4)$ & 100 & \\
\hline LVI & & & 0.549 \\
\hline No & $72(75.8)$ & 96.9 & \\
\hline Yes & $23(24.2)$ & 94.7 & \\
\hline Molecular type & & & 0.309 \\
\hline Luminal A & $9(9.5)$ & 100 & \\
\hline Luminal B & $35(36.8)$ & 96.7 & \\
\hline Non-luminal, HER2+ & $25(26.3)$ & 100 & \\
\hline TNBC & $26(27.4)$ & 90.4 & \\
\hline Histologic grade & & & 0.062 \\
\hline 1,2 & $49(60.5)$ & 100 & \\
\hline 3 & $32(39.5)$ & 92.6 & \\
\hline Resection margin & & & 0.009 \\
\hline Negative & 87 (91.6) & 97.7 & \\
\hline Close or positive & $8(8.4)$ & 87.5 & \\
\hline IMN boost & & & 0.538 \\
\hline No & 83 (87.3) & 96.0 & \\
\hline Yes & $12(12.7)$ & 100 & \\
\hline
\end{tabular}

IMN, internal mammary node; CTX, chemotherapy; LVI, lymphovascular invasion; TNBC, triple-negative breast cancer.

PET-CT, IMN involvement is more frequently observed $[5,9,10]$. This study spotlights the treatment outcomes of initially IMNpositive breast cancer patients, a topic addressed in few prior studies $[3,20,21]$.

Our study revealed that RT alone showed excellent longterm IMN control of initial clinically positive IMNs in breast cancer patients without surgical dissection of IMN (5-year IMN control: 96.4\%). This is concordant with that reported by Veronesi et al. [13], who reported that patients with positive IMNs showed a 5-year IMN control rate of 95\% with appropriate systemic treatment and RT. Zhang et al. [3] also assessed the outcomes of clinically detected IMNs treated with RT, reporting a 5-year IMN control rate of $89 \%$. Historical records show a 5-year locoregional control probability of
93\%-96\% for surgical dissection of IMN [19], confirming that $\mathrm{RT}$ alone is an effective treatment strategy for initially positive IMNs in breast cancer.

DFS and OS were also encouraging considering that most patients had an initial clinical stage of N3, with a 5-year DFS of $70.4 \%$ and 0 S of $84.2 \%$. This is favorable compared to the results of historical studies in which the reported OS was as low as 30\% [22]. Park et al. [20] and Noh et al. [21] also reported that the 5 -year DFS for patients with initial cN3b tumors treated with neoadjuvant chemotherapy, surgery, and RT were 60\% and 60.2\%, respectively. Such improved prognosis in the modern era may be attributed to advanced systemic therapy and precise local therapy.

In our study, IMN involvement was determined based 
only on MR and PET-CT images, and no patient received an IMN biopsy prior to treatment. Thus, we cannot exclude the possibility of false-positive findings. This practice correlates with that in other institutes, wherein IMN involvement is examined with radiologic studies or lymphoscintigraphies. Detection of IMN with $\mathrm{MRI}$ and PET-CT is a noninvasive and reliable method, with sensitivities of $93 \%$ and $85 \%$, respectively, and specificities of $89 \%$ and $90 \%$, respectively $[5,18]$. Lymphoscintigraphy is also a valid method for IMN mapping, with a reported detection rate of 75\%-98\%, which may be improved with an optimized injection site and tracer dose [23]. In contrast, IMN biopsy is technically difficult compared to axillary node biopsy and has complication risks, including pleural breach and damage to the internal mammary vessels [18]. For these reasons, pathological confirmation of IMN is not currently routinely performed in many institutes.

Of the 77 patients who received neoadjuvant chemotherapy, over two-thirds ( $n=52$ ) showed complete resolution of IMN and one-fourth $(n=19)$ showed a partial response. This is consistent with the report by Zhang et al. [3], who revealed that $67 \%$ and $9.8 \%$ of initially IMN-positive patients showed complete and partial IMN responses after neoadjuvant chemotherapy, respectively. Similar normalization rates of IMN and axillary lymph nodes were also reported by Cao et al. [24], who revealed that $64.3 \%$ of patients with initially $\mathrm{N}+$ stage showed N0 stage after neoadjuvant chemotherapy. Previous studies and the ongoing NSABP B-51/RTOG 1304 trial questioned the necessity of adjuvant RT to the regional lymph nodes in breast cancer patients with clinically positive but pathologically negative axillary lymph nodes after neoadjuvant chemotherapy [25]. In this context, the question arises whether we should stratify RT to IMN according to post-neoadjuvant chemotherapy status.

Zhang et al. [3] reported on the institutional outcomes of the response-adapted RT policy of MD Anderson by irradiating the IMN chain with dose discrimination-60 Gy for IMNs normalized after neoadjuvant chemotherapy and 66 Gy for grossly remaining IMNs. The IMN control rate was high in both the $60 \mathrm{~Gy}$ and 66 Gy groups. A study by Yang et al. [26] also retrospectively reviewed data of breast cancer patients with initially apparent IMN to assess the outcomes of IMN boost irradiation. The median dose to the IMN was $62.5 \mathrm{~Gy}$, ranging from 50 to $70.4 \mathrm{~Gy}$. In our study, all IMN failure cases were noted in patients who did not receive IMN boost irradiation. In concordance with our results, Yang et al. [26] reported a potential benefit of IMN boost irradiation (5-year DFS 33.3\% vs. $69.3 \% ; p=0.019$ ), which was more evident when the initial
IMN was larger than $>1 \mathrm{~cm}$. As such, we believe that IMN boost irradiation may be particularly beneficial for patients with an initial IMN size of $\geq 1 \mathrm{~cm}$. However, the optimal boost dose remains undetermined.

No RT-induced severe acute or late toxicities were observed in our study, regardless of IMN boost irradiation. Grade 1 pneumonitis and grade 1 skin reaction were more frequently reported in IMN boost irradiated patients, but this increase in adverse reactions was observed only when the boost was administered in 3D CRT. Despite previous concerns that IMN irradiation may cause adverse effects to the heart and lungs [27-30], the inclusion of IMN in the RT field resulted in a slightly increased incidence of low-grade toxicities and had otherwise no clinical relevance. Previous studies also support the view that irradiation of the regional lymph nodes not only lowers the rates of symptomatic adverse reactions but also minimizes the impact on patient performance $[28,31]$. Modern RT techniques, including deep inspiration breathing hold and IMRT, may further decrease the radiation dose to the heart $[32,33]$. Our institutional data also demonstrated that IMRT decreased grade 1 lung toxicity and skin toxicities of all grades following regional lymph node irradiation. Thus, the potential benefits of IMN boost irradiation might clinically outweigh the risk of adverse effects, given the use of contemporary techniques.

This study has several limitations, partly due to its retrospective design. The small study population and narrow boost dose range hindered complete statistical analysis of IMN irradiation dose on DFS or OS. Moreover, IMN boost irradiation was administered according to the treating physicians' discretion, allowing potential selection bias. In addition, the follow-up time was not sufficient to assess cardiac toxicities, which have been reported to develop as late as 20 years after radiation exposure [29]. Despite these limitations, this study is one of the largest to evaluate IMN boost irradiation and its clinical outcomes. To overcome the study's shortcomings, a multi-center study involving a larger cohort with longterm follow-up is necessary to completely address the clinical benefits and adverse reactions following IMN boost irradiation. Considering that the effect of IMN irradiation was more apparent when measured as DFS than as locoregional recurrence-free survival in previous studies, including the MA.20 [27] and EORTC 22922 trials [34], a focus on DFS may be more meaningful.

In conclusion, the results of this study indicate that RT alone is effective for the local control of initially positive IMNs in breast cancer patients. Moreover, high IMN control with 
limited treatment toxicity can be achieved with IMRT. Recently, the MA.20 [27] and EORTC 22922 trials [34] demonstrated the survival benefit of regional lymph node irradiation including the IMN chain in breast cancer patients without clinical evidence of IMN involvement. In light of such findings, we question the potential benefits of boost irradiation for initially apparent IMNs. Future studies should focus on identifying patients who would most benefit from IMN boost irradiation as well as on optimizing the boost irradiation dose.

\section{Conflict of Interest}

No potential conflict of interest relevant to this article was reported.

\section{References}

1. Oi XW, Du JZ, Tang $P$, et al. Clinical significance of internal mammary lymph node metastasis for breast cancer: analysis of 337 breast cancer patients. Surg Oncol 2018;27:185-91.

2. Hassiotou F, Geddes D. Anatomy of the human mammary gland: current status of knowledge. Clin Anat 2013;26:29-48.

3. Zhang YJ, Oh JL, Whitman GJ, et al. Clinically apparent internal mammary nodal metastasis in patients with advanced breast cancer: incidence and local control. Int J Radiat Oncol Biol Phys 2010;77:1113-9.

4. Klauber-DeMore N, Bevilacqua JL, Van Zee KJ, Borgen P, Cody HS 3rd. Comprehensive review of the management of internal mammary lymph node metastases in breast cancer. J Am Coll Surg 2001;193:547-55.

5. Jochelson MS, Lebron L, Jacobs SS, et al. Detection of internal mammary adenopathy in patients with breast cancer by PET/ CT and MRI. AJR Am J Roentgenol 2015;205:899-904.

6. Veronesi U, Cascinelli N, Bufalino R, et al. Risk of internal mammary lymph node metastases and its relevance on prognosis of breast cancer patients. Ann Surg 1983;198:6814.

7. Cody HS 3rd, Urban JA. Internal mammary node status: a major prognosticator in axillary node-negative breast cancer. Ann Surg Oncol 1995;2:32-7.

8. Noguchi M, Ohta N, Thomas M, Kitagawa H, Miyazaki I. Risk of internal mammary lymph node metastases and its prognostic value in breast cancer patients. J Surg Oncol 1993;52:26-30.

9. Davidson T, Ben-David M, Galper S, et al. Use of (18)F-FDG PET-CT imaging to determine internal mammary lymph node location for radiation therapy treatment planning in breast cancer patients. Pract Radiat Oncol 2017;7:373-81.
10. Seo MJ, Lee JJ, Kim HO, et al. Detection of internal mammary lymph node metastasis with (18)F-fluorodeoxyglucose positron emission tomography/computed tomography in patients with stage III breast cancer. Eur J Nucl Med Mol Imaging 2014;41:438-45.

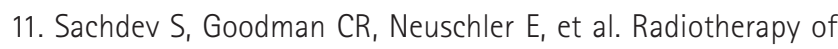
$\mathrm{MRI}$-detected involved internal mammary lymph nodes in breast cancer. Radiat Oncol 2017;12:199.

12. Urban JA. Radical excision of the chest wall for mammary cancer. Cancer 1951;4:1263-85.

13. Veronesi $U$, Arnone $P$, Veronesi $P$, et al. The value of radiotherapy on metastatic internal mammary nodes in breast cancer: results on a large series. Ann Oncol 2008;19:1553-60.

14. Veronesi U, Marubini E, Mariani L, Valagussa P, Zucali R. The dissection of internal mammary nodes does not improve the survival of breast cancer patients: 30 -year results of a randomised trial. Eur J Cancer 1999;35:1320-5.

15. Pardolesi A, Bertolaccini L, Brandolini J, Solli P. Robotic internal mammary lymphadenectomy: another possible minimally invasive approach to sampling lymph nodes in breast cancer patients. J Vis Surg 2018;4:71.

16. Goldhirsch A, Winer EP, Coates AS, et al. Personalizing the treatment of women with early breast cancer: highlights of the St Gallen International Expert Consensus on the Primary Therapy of Early Breast Cancer 2013. Ann Oncol 2013;24:2206-23.

17. Common Terminology Criteria for Adverse Events (CTCAE) version 4.0 [Internet]. Bethesda, MD: US National Cancer Institute; 2010 [cited 2019 Feb 27]. Available from: https://evs.nci.nih.gov/ftp1/CTCAE/CTCAE_4.03/ CTCAE_4.03_2010-06-14_QuickReference_5x7.pdf.

18. Chen RC, Lin NU, Golshan M, Harris JR, Bellon JR. Internal mammary nodes in breast cancer: diagnosis and implications for patient management: a systematic review. J Clin Oncol 2008;26:4981-9.

19. Veronesi U, Valagussa P. Inefficacy of internal mammary nodes dissection in breast cancer surgery. Cancer 1981;47:170-5.

20. Park HJ, Shin KH, Cho KH, et al. Outcomes of positron emission tomography-staged clinical N3 breast cancer treated with neoadjuvant chemotherapy, surgery, and radiotherapy. Int J Radiat Oncol Biol Phys 2011;81:e689-95.

21. Noh JM, Kim KH, Park W, et al. Prognostic significance of nodal involvement region in clinical stage IIlc breast cancer patients who received primary systemic treatment, surgery, and radiotherapy. Breast 2015;24:637-41.

22. Veronesi $U$, Cascinelli N, Greco $M$, et al. Prognosis of breast cancer patients after mastectomy and dissection of internal 
mammary nodes. Ann Surg 1985;202:702-7.

23. Valdes-Olmos RA, Jansen $L$, Hoefnagel $C A$, et al. Evaluation of mammary lymphoscintigraphy by a single intratumoral injection for sentinel node identification. J Nucl Med 2000;41:1500-6.

24. Cao XS, Li HJ, Cong BB, et al. Axillary and internal mammary sentinel lymph node biopsy in breast cancer after neoadjuvant chemotherapy. Oncotarget 2016;7:74074-81.

25. Liu J, Mao K, Jiang $S$, et al. The role of postmastectomy radiotherapy in clinically node-positive, stage II-III breast cancer patients with pathological negative nodes after neoadjuvant chemotherapy: an analysis from the NCDB. Oncotarget 2016;7:24848-59.

26. Yang $\mathrm{K}$, Choi DH, Kim H, et al. Outcome of radiotherapy in patients with internal mammary lymph node metastasis from breast cancer. In: Proceedings of 2018 KOSRO Annual Meeting; 2018 Oct 12; Seoul, Korea.

27. Whelan TJ, Olivotto IA, Parulekar WR, et al. Regional nodal irradiation in early-stage breast cancer. N Engl J Med 2015;373:307-16.

28. Matzinger 0 , Heimsoth I, Poortmans $P$, et al. Toxicity at three years with and without irradiation of the internal mammary and medial supraclavicular lymph node chain in stage I to III breast cancer (EORTC trial 22922/10925). Acta Oncol 2010;49:24-34

29. Darby SC, Ewertz M, McGale P, et al. Risk of ischemic heart disease in women after radiotherapy for breast cancer. $N$ Engl J Med 2013;368:987-98.

30. Chargari $C$, Castadot $P$, Macdermed $D$, et al. Internal mammary lymph node irradiation contributes to heart dose in breast cancer. Med Dosim 2010;35(3):163-8.

31. Chung Y, Yoon HI, Kim YB, Ahn SK, Keum KC, Suh CO. Radiation pneumonitis in breast cancer patients who received radiotherapy using the partially wide tangent technique after breast conserving surgery. J Breast Cancer 2012;15:337-43.

32. Chang JS, Park W, Kim YB, et al. Long-term survival outcomes following internal mammary node irradiation in stage II-III breast cancer: results of a large retrospective study with 12year follow-up. Int J Radiat Oncol Biol Phys 2013;86:867-72.

33. Rudat $V$, Alaradi AA, Mohamed A, Ai-Yahya K, Altuwaijri S Tangential beam IMRT versus tangential beam 3D-CRT of the chest wall in postmastectomy breast cancer patients: a dosimetric comparison. Radiat Oncol 2011;6:26.

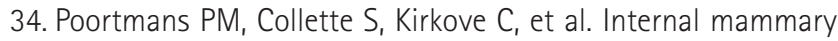
and medial supraclavicular irradiation in breast cancer. $\mathrm{N}$ Engl J Med 2015;373:317-27. 